\title{
ESTRATEGIAS PARA LA GESTIÓN DE LOS PAISAJES \\ RURALES DISPERSOS. EL CASO DEL PARQUE LOCAL DE INTERÉS SUPRAMUNICIPAL DE LAS TIERRAS DE LOS NAVIGLI' (ITALIA) \\ Marcello Magoni*
}

Paisaje Tierra de Navigli

Fuente: Suministrada por el autor

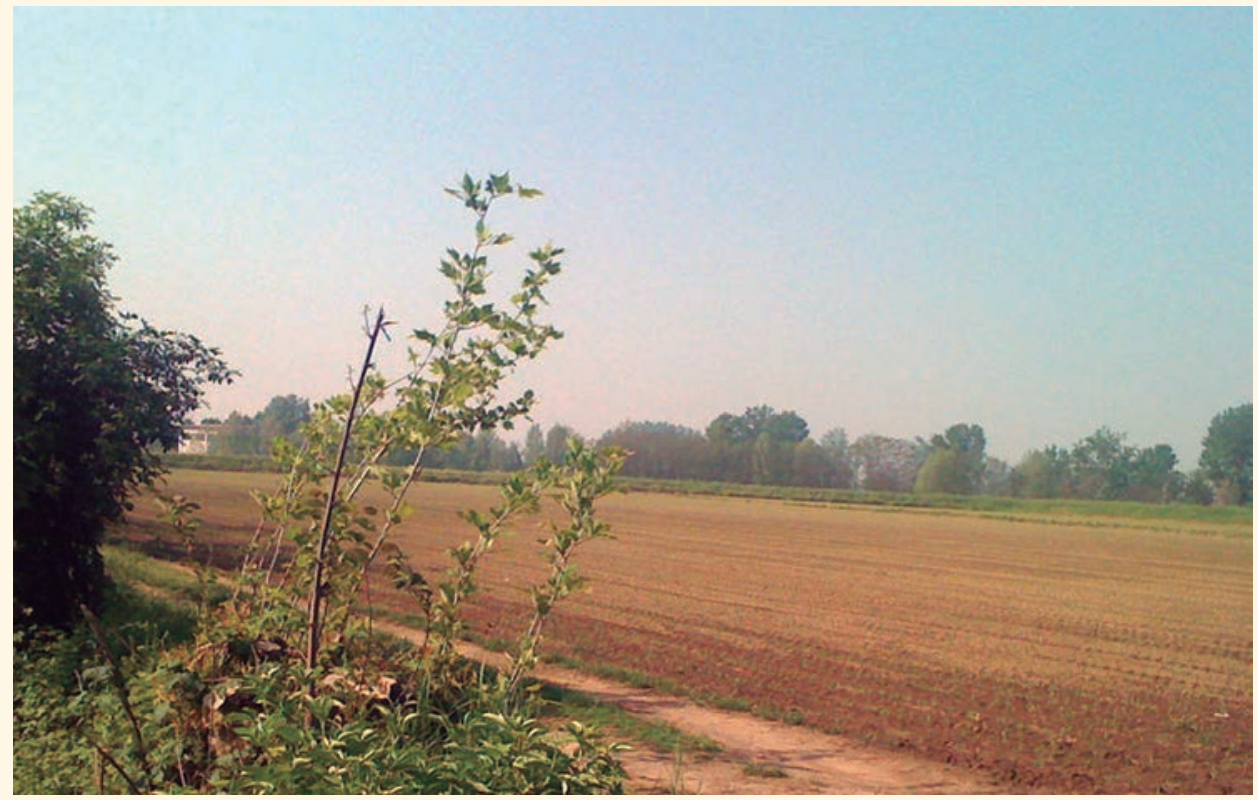

\section{RESUMEN'}

Con la aprobación de la Convención Europea del Paisaje, en 2000, se abrió una nueva etapa de la planificación territorial en Europa gracias a la introducción de algunos de los principios innovadores como la extensión del concepto de paisaje a todo el territorio, la introducción del tema de diseño de nuevos paisajes y la participación de la población para valorar la calidad de ellos. Por otra parte, desde hace algunos decenios, uno de los temas de mayor interés en la planificación territorial está relacionado con la salvaguardia de las áreas agrícolas ante la expansión urbana y la aparición de nuevos desarrollos, en la que la presencia de vastas áreas agrícolas es un elemento territorial indispensable para reconfigurar la ciudad en términos de sostenibilidad. Este artículo expone los principales aspectos de una experiencia innovadora de planificación, relacionados con la creación de un área destinada a acoger un parque de carácter supramunicipal -el Parque Local de interés intermunicipal de las Tierras de los Navigli -en un ámbito rural del Norte de Italia, donde se han hecho converger diferentes valores e intereses, ya sean de índole cultural, paisajístico-ambiental o disciplinar. Además, en la construcción del proyecto de paisaje se adoptó un enfoque coherente con la Convención Europea del Paisaje, ya que se le dio a todo el territorio la valencia de paisaje con base en sus dinámicas socio-económicas y espaciales.

\section{PALABRAS CLAVE}

Gestión paisaje rural, Paisaje rural, Parque local supramunicipal, Navigli

Nota del traductor: El término "Navigli" (plural de "Naviglio") significa navegable en italiano pero una traducción literal en español sería bastante forzada. Por lo tanto, se recomienda al lector evocar con la palabra "Navigli" una serie de canales, iniciados por los residentes de Milán y sus alrededores en el siglo XII que, según los historiadores, sirvieron en primera instancia para defender la ciudad y más tarde para proporcionar una ruta de comercio para el transporte fluvial. Como dato importante, vale la pena anotar que en el Siglo XV, Leonardo da Vinci trabajó en uno de los canales de los Navigli, exactamente en un sistema de exclusas para facilitar la navegación entre dichos canales. Fuente: www.navigli.net 


\section{STRATEGIE PER LA GESTIONE DEI PAESAGGI RURALI DIFFUSI. IL CASO DEL PARCO LOCALE DI INTERESSE SOVRACOMUNALE DELLE TERRE DEI NAVIGLI (ITALIA).}

Marcello Magoni*

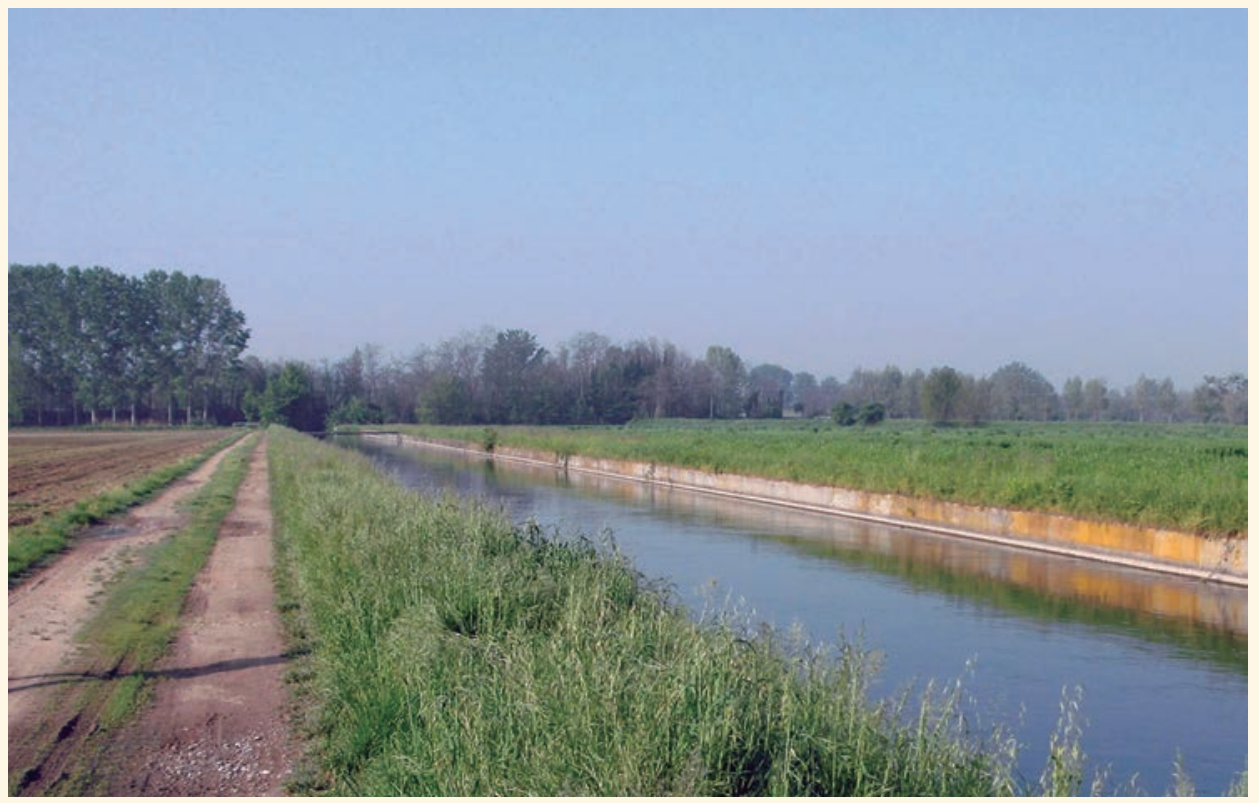

\begin{abstract}
Con l'approvazione nel 2000 della Convenzione Europea del Paesaggio, si è aperta una nuova stagione della pianificazione territoriale in Europa dovuta all'introduzione di alcuni principi innovativi quali l'estensione del concetto di paesaggio a tutto il territorio, l'introduzione del tema della progettazione di nuovi paesaggi e il coinvolgimento della popolazione nella valutazione della loro qualità. D'altra parte, da alcuni decenni uno dei temi di maggior interesse per la pianificazione urbanistica e territoriale riguarda la tutela delle aree agricole dalle espansioni urbane e insediative, essendo la presenza di vaste aree agricole un elemento territoriale indispensabile per riconfigurare le città in senso sostenibile. L'articolo espone i principali aspetti di un'esperienza innovativa di pianificazione legata all'istituzione di un'area a parco di carattere sovracomunale - il Parco locale di interesse sovracomunale delle Terre dei Navigli - in un ambito rurale del Nord Italia, dove sono stati fatti convergere differenti valori e interessi, da quelli culturali a quelli paesistico-ambientali a quelli disciplinari. Inoltre, nella costruzione del progetto di paesaggio è stato adottato un approccio coerente con la Convenzione Europea del Paesaggio, poiché si è estesa a tutto il territorio la valenza di paesaggio considerata nelle sue dinamiche socio-economiche e spaziali.
\end{abstract}

\section{PAROLE CHIAVE}

Gertione dei paesaggi rurali, paesaggio rurale, Navigli, Parco locale sovracomunale
Recibido: 27 de enero de 2011 Aprobado: 27 de abril de 2011

Vista canal Tierra Navigli

Fuente: Suministrada por el autor 


\section{INTRODUCCIÓN}

Con la aprobación de la Convención Europea del Paisaje, sesionada en Florencia en 2000 y firmada por varios países europeos, entre ellos Italia, se abrió una nueva etapa de la planificación paisajística y territorial mediante la introducción de algunos de los principios innovadores cuya aplicación ha exigido, y exige todavía, avances importantes en las disciplinas de planificación. Esto se debe a que, al declarar su interés por la totalidad de un territorio que incluye tanto "paisajes excepcionales" como "paisajes de la vida cotidiana y paisajes degradados", el Convenio apoya el tema de la protección y conservación activa, la rehabilitación y la formación de nuevos paisajes.

Además, se plantea como un factor determinante para evaluar la calidad del paisaje, la participación de la población de un territorio, con la consiguiente necesidad de activar una confrontación con los "externos", o sea, personas no residentes en la zona, pero con buen conocimiento del paisaje allí presente, y los "internos", o sea, los residentes en la zona que actúan directa o indirectamente sobre el paisaje y que a menudo no lo conocen. Por otro lado, desde hace algunas décadas uno de los temas más interesantes para la planificación urbanística y territorial tiene que ver con la protección de las zonas agrícolas de la expansión urbana y de los nuevos asentamientos, la presencia de grandes extensiones agrícolas es un elemento territorial indispensable para volver a configurar las ciudades en forma sostenible. ${ }^{2}$ La reconsideración del papel de los territorios rurales, ya sean residuales, en zonas periféricas o áreas agrícolas de elevada productividad, se abre a múltiples facetas de la planificación y del diseño del paisaje, con relación a las diversas dinámicas, las relacionadas con los asentamientos como las de índole social y económicas que requieren la activación de políticas, estrategias y proyecciones diferenciadas

La oportunidad de establecer, en una zona rural, un área destinada a un parque de carácter intermunicipal -el parque local de intereses intermunicipales de las Tierras de los Navigli (PLIS-TdN)- ha hecho converger diferentes valores e intereses (culturales, paisajísticos, ambientales y disciplinarios) hacia la realización de una avanzada experiencia de planificación y de tutela territorial. De hecho, en la construcción del proyecto de paisaje se ha adoptado un enfoque coherente con la Convención, al extender a todo el territorio el valor del paisaje en sus dinámicas socio-económicas y ambientales e involucrada la población local.

\section{El Convenio Europeo del Paisaje y la gestión de los paisajes rurales}

Con la firma del Convenio Europeo del Paisaje, en Italia y en muchos países europeos, el paisaje se ha convertido en objeto de gran preocupación para el público en general, los ingenieros y los administradores públicos. Además, con la aprobación en 2004 en Italia del Código de Patrimonio Cultural y del Paisaje, los municipios están llamados a adecuar los planes urbanísticos a los nuevos planes paisajísticos y a evaluar la compatibilidad paisajística de las intervenciones en áreas sometidas a restricciones (vínculos), los cuales afectan grandes áreas del territorio.

De esta manera, se experimentan los instrumentos operativos y culturales más idóneos para responder a las numerosas implicaciones de esta gran "demanda de paisaje"

2 Los temas de los paisajes agrícolas y de sus relaciones con la ciudad dispersa, constituyen argumentos centrales del actual debate científico europeo. Consultar, específicamente, los escritos de Donadieu sobre el papel y la multifuncionalidad del territorio rural y los de Peano sobre el paisaje agrícola y de los parques. 
La ampliación de las políticas paisajistas a todo el territorio incentiva una mayor atención por las actividades de gestión y diseño del paisaje, sobre todo de los ordinarios y degradados. El diseño del paisaje tiende a elaborar acciones de transformación funcional, ya sea por las exigencias evolutivas del paisaje como por las de protección, al insertar nuevas formas de manera coherente con el contexto que respetan tanto lo que se ha heredado como las instancias de la contemporaneidad.

Las premisas fundamentales para el proyecto y la gestión del paisaje son la comprensión de la evolución de las formas paisajísticas -desde la individuación de los elementos que "constituyen" el paisaje, que describen las dinámicas que condujeron a la actual situación y aquellas futuras que puedan afectar que indican además, los individuos que de manera directa o indirecta pueden contribuir a su gestión y transformación- y la lectura del estado de hecho.

Son profundizadas las modalidades de transferencia de las indicaciones, desde una gran escala a la escala local y de intervención, porque la planificación urbanística incorpora, en general, el sistema de restricciones paisajistas pero no desarrolla los proyectos. Luego, se ponen en consideración las diferentes relaciones entre escalas, sectores y campos disciplinarios para lograr una mayor operatividad del proyecto del paisaje y tratar de traducir las directrices de los planes de amplia cobertura en sistemas interrelacionados de buenas prácticas y de instrumentos normativos más detallados.

En el plano internacional es prometedor el debate sobre las experiencias que se reconocen en "Landscape Urbanism" (C. Waldheim, 2006), donde se desarrollaron los métodos y criterios de interpretación y evaluación de las dinámicas territoriales para guiar la transformación del paisaje y mantener vivos los valores de identidad de sitios específicos y/o ayudar a construir otras nuevas. En este sentido, la representación y la comunicación de los valores del paisaje deben participar expertos y los no expertos en el paisaje para lograr el reconocimiento social y la imagen de los lugares de su contexto.

Para facilitar la tutela de las áreas en los nuevos escenarios de desarrollo territorial, se propone el concepto de redes multifuncionales, o sea, un sistema de territorios conectados, capaces de proporcionar un conjunto integrado de servicios como espacios abiertos, la tutela de la biodiversidad, la calidad ambiental, la producción de alimentos y la valorización de la identidad local. Estas redes se consideran como infraestructuras de un nuevo urbanismo funcional para responder a múltiples demandas a través de la construcción de tejidos territoriales que reconecten lugares y fragmentos de territorio, de intervenciones que dan un nuevo significado a porciones de la ciudad en expansión, de proyectos de renovación ambiental y de producciones de nuevos paisajes.

Componentes centrales de las redes multifuncionales son las redes ecológicas, que a menudo interesan las áreas agrícolas y se configuran como "infraestructuras" naturales a través de las cuales se recuperan y se remiendan todos los ambientes dispersos que han mantenido una estructura que a pesar de ser residual continúa a ser originariamente natural. Estas redes pueden usufructuar de los fondos europeos para "mandar a descansar" los terrenos agrícolas, reducir el impacto ambiental de las actividades agrícolas y, de esta manera, conseguir una mejor gestión ambiental del territorio mediante la introducción de intervenciones en un diseño más general e integrado que garantice la continuidad en el tiempo de las funciones allí presentes. 


\section{El parque local de interés supramunicipal como instrumento de protección de los paisajes rurales lombardos}

El parque local de interés supramunicipal es un instrumento de protección del territorio, exclusivo de la Lombardía, introducido en 1983 por la ley regional de áreas protegidas, que tiene como propósito la planificación, la gestión de la protección y de la renovación de los territorios en modo participativo. De hecho, los PLIS han sido instaurados por otras administraciones municipales que comparten la voluntad de proteger y valorizar una parte de su territorio desde el punto de vista paisajístico y ambiental y son la ocasión para propiciar una participación activa del voluntariado y del asociacionismo local, al acercar la población al ambiente y al paisaje.

Las principales áreas interesadas por los PLIS son los elementos de interés secundario de las redes ecológicas regionales y provinciales, elementos que, de una parte unen los componentes primarios de las redes ecológicas $y$, por otra parte, van a integrar los sistemas del verde a escala urbana.

Los PLIS contribuyen en mayor medida a proteger el paisaje rural respecto a los planes urbanísticos porque disponen de instrumentos jurídicos y económicos más idóneos para orientar las transformaciones de un paisaje que está estrechamente ligado al tipo de actividad agrícola que allí se desarrolla. Estos tienen una mayor facilidad para soportar en el tiempo modelos innovadores de producción agrícola, capaces de dar respuesta a las dinámicas económicas a largo plazo y a las exigencias de calidad territorial y ambiental. Además, en los contextos periurbanos, desempeñan una función reguladora del crecimiento de los asentamientos, desordenado por lo general, y de contención de lo urbanizado, pues, forman cinturones verdes que evitan que se fusionen las poblaciones pertenecientes a diferentes municipios.

En el 2009, el número instituido de los PLIS había alcanzado 87 unidades, que llegaba a interesar más del $3 \%$ del territorio total regional, es decir, $800 \mathrm{~km}$. Los 87 PLIS se suman a un parque nacional, a 24 parques regionales, a 64 reservas naturales y a 31 monumentos naturales que protegen más del $21 \%$ del territorio regional. El tipo de instrumentos a los cuales han sido sometidas las áreas naturales lombardas es en función de carácter del territorio, el cual, en Lombardía está muy diversificado en sentido norte-sur pues va desde alturas que llegan a los $4.000 \mathrm{~m}$ de la cadena montañosa de los Alpes, la cual representa una de las más importantes áreas naturales europeas por extensión y riqueza de biodiversidad, hasta la franja pre alpina y, por tanto, a la zona de la llanura Padana que llega hasta el río Po. Estas tres franjas están conectadas entre sí por numerosos ríos que atraviesan el territorio en sentido norte-sur y cuyas aguas confluyen totalmente el río Po.

\section{Las características territoriales del PLIS de las Tierras de los Canales (Navigli)}

Tierras de los Navigli es el nombre que se le atribuye a un grupo de II Municipios situado en un fragmento de la llanura lombarda en la provincia de Cremona, los cuales, han desarrollado una planificación compartida del propio territorio, interpretada en II planos urbanísticos integrados. La denominación de las Tierras de los Navigli ha incluido los canales navegables y la red hidrográfica como matriz, simbólica y territorial, de un desarrollo durable y capaz de valorizar el capital social y de ponerse en relación con las "largas redes" del desarrollo interregional. 
EI PLIS-TdN constituye uno de los proyectos cualitativos para las Tierras de los Navigli ya que además de interesar 49 de los $183 \mathrm{~km}^{2}$ de la superficie del área, equivalentes a aproximadamente el $27 \%$ (Figura I), genera economías y relaciones locales innovadoras que podrán ser difundidas en otros territorios.

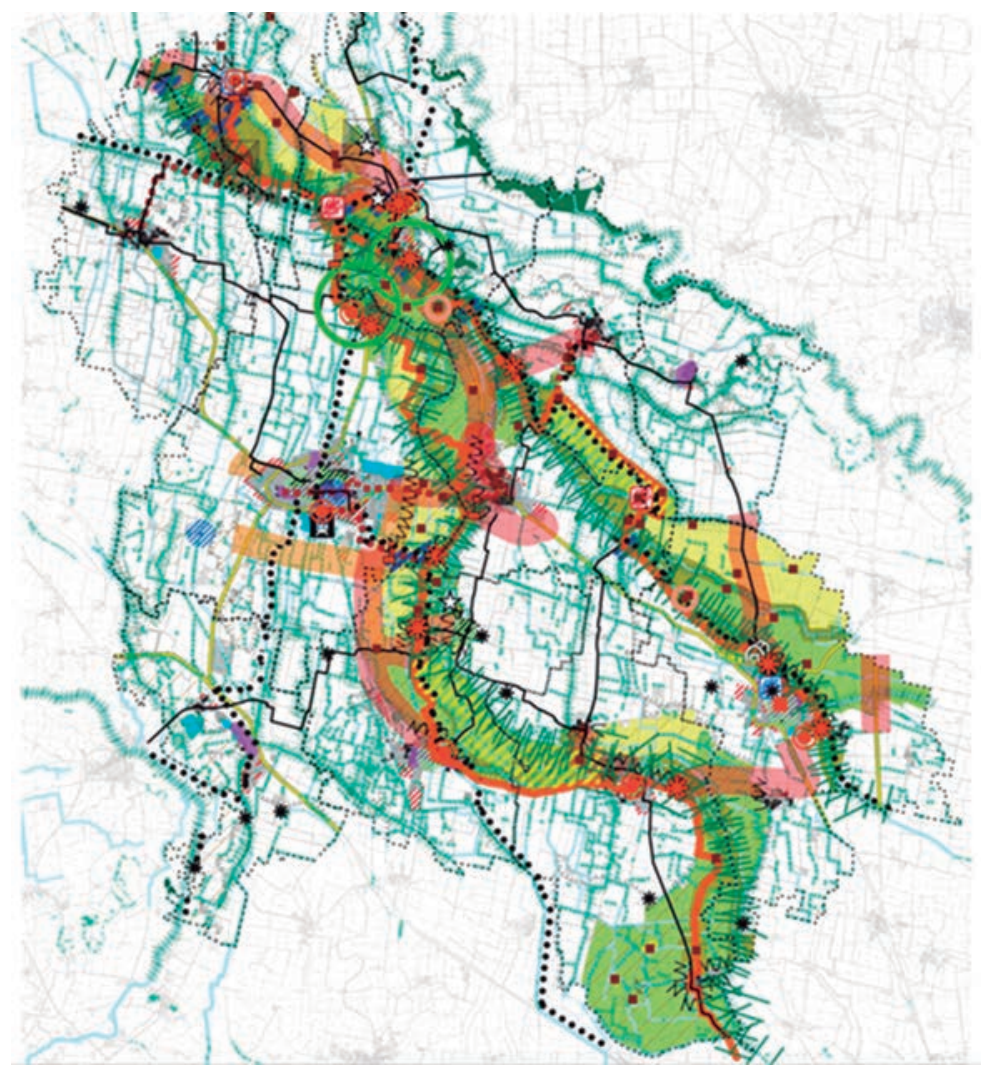

El territorio de las Tierras de los Navigli, aunque presente una urbanización contenida, es difusamente artificial -con excepción de los ámbitos del valle fluvial del Oglio y del sistema de los Navigli- no presenta significativos ámbitos naturales o paranaturales. En este territorio no hay fuertes presiones de nuevos asentamientos) ni de tipo residencial ni productivo.

El territorio del PLIS-TdN, está constituido prevalentemente por un valle fluvial aislado, llamado del Morbasco, que atraviesa las Tierras de los Navigli de norte a sur, al cual se añade una amplia banda (grupo de franja, área) de canales que salen del valle del Morbasco y prosiguen en dirección al sur.

EI PLIS-TdN, toca (interesa) un componente de la red ecológica provincial que se pone en posición intermedia y paralela a dos componentes de la red ecológica regional, constituidos por los valles fluviales del río Oglio y del río Serio. Mientras los dos valles fluviales son caracterizados por la presencia de vastas áreas naturales de valor, el territorio del PLIS-TdN está caracterizado sobre todo por actividades agrícolas. Por tanto, el actual paisaje del PLIS-TdN es el producto de dinámicas económicas y de técnicas agrícolas que condicionan fuertemente la evolución y las formas, tanto de las edificaciones como de los espacios abiertos.

En el pasado el territorio se estructuraba a través de grandes propiedades en torno a las cascine (antiguos cortijos o granjas), las cuales eran lugares de residencia, de vida de las
Figura I - El escenario de aplicación del PLIS-TdN

Fuente: PGT-I de las Tierras de los Navigli. Suministrado por el autor 
Figura 2 - Esquematización del proceso de evolución del paisaje de la llanura padana.

Fuente: Maurizio Guido Paoletti and Giovann Giorgio Lorenzoni, Agroecology patterns in Northeastern Italy, Agriculture, Ecosystems \& Environment. comunidades (aquí vivían los conductores y algunos asalariados que eran hospedados como trabajadores estacionales) y de las primeras transformaciones de productos agrícolas. La estructura de los campos, delimitada por la presencia de setos e hileras de arboles, incluía la presencia de prados perennes, praderas de inundación, campos de cereal y maíz. Con el tiempo, en el área se desarrolla la cadena productiva de lácteos caseros, con criaderos situados en las antiguas granjas.

En los primeros años del siglo $\mathrm{XX}$, las producciones agrícolas se integran con las producciones industriales, que introducen cultivos conectados a ellas, como el cáñamo y el lino y se difunde notablemente el cultivo del maíz. Los setos altos se presentan a lo largo de los caminos de las granjas, las orillas de los canales y, a lo largo de los bordes de los grandes campos de regadío, y rescatan la imagen de una llanura llena de árboles. Estos se caracterizan por la estrecha conexión entre especie, forma y funciones desempeñadas, como la división de los campos, el suministro de madera para construcción, la producción de frutas y otros, para definir de esta manera, un paisaje típico, complejo y homogéneo.

Con el tiempo, las funciones de los setos altos se reducen más hasta asumir actualmente, sobre todo, valencias de índole ecológica (ligadas al mantenimiento de la biodiversidad) y paisajística (Figura 2). El riesgo actual es el de perder el sistema de setos bajos y altos, que constituye un elemento de grandísimo valor paisajístico, capaz de testimoniar y conservar la memoria de las comunidades campesinas y de las modalidades con las cuales ella se había asentado en el territorio y lo había vivido y transformado.

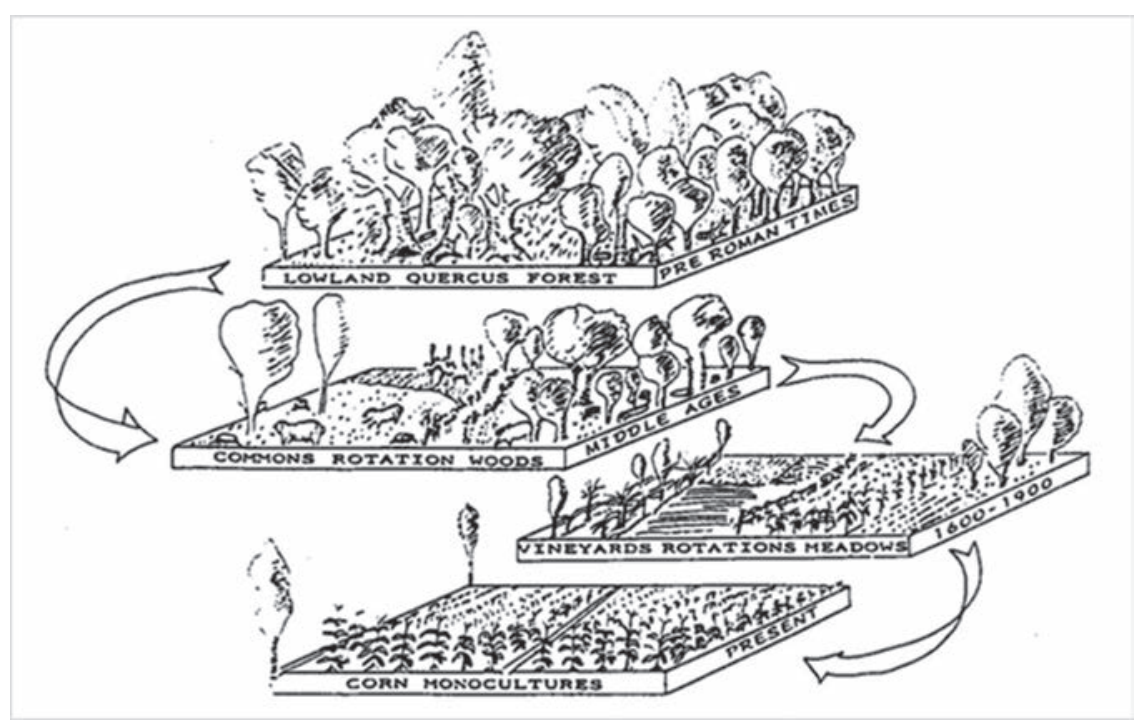

Las causas que han determinado el proceso de simplificación del paisaje rural se ubican en la mutación de las técnicas y de los procesos productivos agrícolas: progresiva mecanización agrícola, contínua regularización de los campos, radicales transformaciones de los procesos y técnicas de zootecnia, recuperación de superficies no cultivadas y reducción de las sombras sobre los cultivos.

El paisaje actual (Fotografía I) presenta todavía una buena complejidad y diferenciación que aumenta a gran medida en proximidades de la extensa y densa red de canales de origen medieval que atraviesa el territorio. En muchas partes del territorio del PLIS-TdN se mantuvo la forma, las dimensiones y la orientación de la trama agrícola, precendentemente realizada con las seculares recuperaciones de los terrenos y los sistemas de irrigación dirigidos por institutos y entes religiosos. 
Hay una difundida presencia de valiosos núcleos de cascine, a menudo poco utilizadas y en fase de degradación, en el que su reutilización, ampliación y reorganización han sido a menudo concebidas en manera no armónica, con cánones edilicios poco propensos al respeto de las tradiciones. Paralelamente, se tuvo una notable difusión de nuevos tipos de construcción, destinados a la agricultura intensiva de ganado y de plantas al servicio de la producción agrícola que por materiales y colores contrastan fuertemente con el paisaje rural tradicional.

Los canales de irrigación son ambientes ricos en diversidad que favorecen el mantenimiento de los ecosistemas acuáticos y conectan diferentes biotipos acuáticos, a veces muy distantes entre ellos, ya sean de tipo natural o artificial. Además, la red hídrica está caracterizada por la presencia de nudos hidráulicos de elevada calidad paisajística e histórico-arquitectónica y por numerosas obras hidráulicas de gran interés, como los molinos o las esclusas. (Fotografías 2 y 3 )
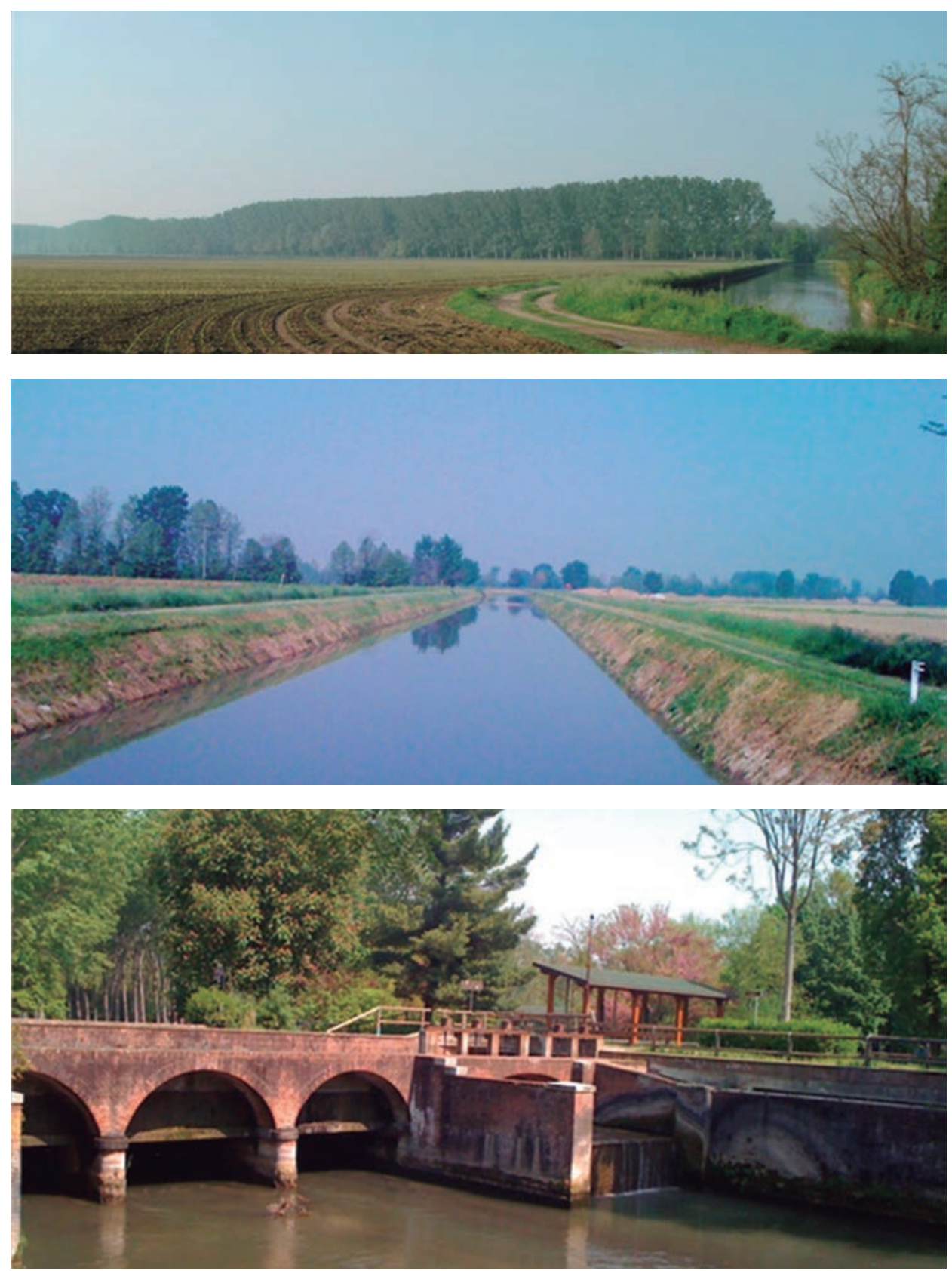

Fotografía I - Vista de una parte del paisaje típico de las Tierras de los Navigli. Suministrado por el autor

Fotografía 2- Vista de algunos canales de las Tierras de los Navigli. Suministrado por el autor

Fotografía 3 - Vista de un nodo hidráulico de las Tierras de los Navigli. Suministrado por el autor 
Además de los tradicionales análisis de los sistemas territoriales, el cuadro cognoscitivo de las Tierras de los Navigli, ha profundizado los aspectos históricos, sociales y perceptivos. La lectura histórica de las transformaciones ha sido efectuada para reconocer los elementos y las estructuras del paisaje y sus relaciones, individuando la permanencia de los principales elementos naturales (los bordes de la escarpada, manantiales, zonas de bosques y demás) y antrópicos (granjas, redes viarias rurales, redes hídricas artificiales, red de carreteras y otros.).

La lectura de los aspectos sociales consistió en la identificación, a través de una comparación de las indicaciones de los agricultores, administradores públicos y académicos del paisaje, de los elementos de reconocimiento e identidad del paisaje local. Fue así como fueron identificados importantes lugares, como los monumentos, como también elementos menores a los que los habitantes prestan atención como las ermitas, por ejemplo.

La lectura de los aspectos de percepción tiene que ver con los factores y fenómenos que informan el juicio estético, privilegiando los juicios sobre situaciones de homogeneidad / heterogeneidad con respecto a la coherencia con la imagen tradicional del paisaje rural y de acuerdo a la presencia de fenómenos de degradación o de incoherencia, como las fábricas y los edificios rurales altamente disonantes con las formas y los materiales tradicionales.

\section{El enfoque de la planificación y de los objetivos para el PLIS-TdN}

El enfoque adoptado en la planificación, tanto del territorio de las Tierras de los Navigli, como del territorio del PLIS-TdN es de tipo estratégico y está orientado a la valorización de las potencialidades y de las dotaciones territoriales con el fin de conseguir una mejor calidad del desarrollo de los asentamientos y del vivir. Para el territorio de la Tierra de los Navigli ha sido individuada una visión ${ }^{3}$ sobre la base de tres principios de desarrollo territorial compartidos por II municipios:

- Conseguir una elevada calidad urbana y territorial, entendida como calidad de los lugares urbanos y no urbanos, de los valores paisajísticos y ambientales, de los servicios públicos y colectivos erogados, de las oportunidades ocupacionales y de emprendimiento, de la valorización del capital social.

- Favorecer la innovación y la competitividad del área, entendidas como el refuerzo de la capacidad de poner en marcha procesos territoriales innovadores y competitivos, como la dotación de servicios de calidad para el sistema productivo (formación, gestión, investigación y desarrollo, comunicación digital y demás), adecuados niveles de accesibilidad, estructuras y redes tecnológicas avanzadas.

- Mantener la complementariedad y la solidaridad territorial, entendidas como la capacidad de llegar a un desarrollo de la comunidad fundado sobre valores de solidaridad y de compartir los aspectos positivos y negativos que las dinámicas de naturaleza exógena inducen al progreso (desarrollo local).

La visión identifica en el habitar, en lugares agradables y tranquilos con buenas oportunidades socio-culturales y económicas, la clave de referencia de las previsiones del plan. En particular, este modo de habitar requiere la construcción de un territorio que se caracterice por una reducida cantidad de asentamientos, de paisajes urbanos, agrícolas y

3 La visión es una idea intencional de futuro, cuya construcción social se mide con los recursos a disposición y con las aspiraciones de los sujetos que viven y actúan en un territorio. Su función es la de construir una idea de desarrollo territorial a largo plazo con el que se puedan orientar las previsiones, los proyectos y las intervenciones que se propondrán. 
fluviales agradables, en los cuales, las distintas funciones se lleven a cabo en contextos (edificios residenciales e industriales, espacios públicos urbanos, servicios, ambientes) de buena calidad, con una satisfactoria cohesión y con una elevada seguridad respecto a los riesgos territoriales (inundaciones, accidentes de tránsito e industriales). Además, esta manera de habitar se apoya en una buena cohesión territorial interna, caracterizada por una satisfactoria movilidad basada en una modalidad de transporte con bajo impacto ambiental y en una conexión suficiente con red vehicular y ferroviaria a nivel regional y nacional.

Las previsiones del plan han sido, por lo tanto, organizadas respecto a dos recorridos. El primero desde la visión lleva a los objetivos del plan y, por tanto, a las estrategias y a las acciones hechas para conseguirlos. El segundo consiste en la construcción del escenario del plan, que restituye el cuadro de las decisiones comunes a todo el territorio de la $\mathrm{TdN}$ y es el resultado del proceso de integración de los objetivos, de las estrategias y de las acciones individuadas con anterioridad. Esto constituye una referencia de la dimensión estratégica del PGT-I y representa el diseño territorial, modificable en el tiempo, hacia el cual las Tierras de los Navigli quieren proceder.

Al respecto, el territorio rural ha sido asumido como el elemento estructural de las Tierras de los Navigli, para lo cual, han sido adaptadas decisiones para los asentamientos y las infraestructuras lo más compactas posibles y con dimensiones contenidas para preservar dichas actividades, la continuidad del espacio rural y así prever la valorización del paisaje rural y el desarrollo de las actividades agrícolas orientadas a la sostenibilidad ambiental y a la calidad territorial.

El proyecto de red ecológica intermunicipal y la institución del PLIS-TdN constituyen los dos instrumentos principales para mejorar la calidad del paisaje rural, la dimensión y la conectividad entre áreas naturales.

La construcción de la red ecológica integra varios objetivos de plan, como el mejoramiento del paisaje (rural, de los ambientes periurbanos y urbanos), el mejoramiento de la facilidad de uso y la accesibilidad a los paisajes de los ámbitos rurales y naturales (redes y recorridos ciclopeatonales conectados a las redes ecológicas), la valorización de los lugares y elementos del paisaje de los espacios abiertos.

La creación del PLIS-TdN propende por:

- Mejorar una calidad difundida del paisaje a través del mantenimiento de los valores constitutivos y de las formas naturales y antrópicas, la recuperación de los valores preexistentes aunque estén degradados o comprometidos y la creación de nuevos valores paisajísticos coherentes e integrados.

- Potenciar y mejorar las funciones y los componentes ecológicos.

- Desarrollar una agricultura multifuncional en armonía con la protección del patrimonio histórico-cultural y ambiental y en coherencia con las políticas agrícolas comunitarias, siempre más orientadas hacia la reducción de los subsidios para las producciones de carácter cuantitativo y su reorientación hacia acciones de sostenibilidad ambiental y de producción de servicios ecológicos ambientales.

El proyecto de paisaje para el PLIS-TdN ha sido elaborado bajo los siguientes criterios:

- Las intervenciones sobre el paisaje son vistas no tanto en relación a los caracteres originarios de imposible definición o significado sino en relación a aquellos que vienen 
definidos actualmente como los caracteres de identidad y de reconocimiento del "paisaje de las Tierras de los Navigli" y que pueden ser valorados a través de conceptos como integridad, cumplimiento y totalidad

- Las intervenciones parten del conocimiento cuidadoso de los lugares y se preocupan por introducir nuevas formas en modo coherente con el contexto y respetan tanto lo heredado, como las instancias de la contemporaneidad;

- Las intervenciones deben dar identidad y servicios de calidad a las poblaciones asentadas y, al mismo tiempo, deben activar formas de atracción del contexto local de parte de poblaciones externas, con el propósito de recuperar recursos adecuados a la recuperación y a la valorización de los bienes con riesgo de degradación.

\section{Las estrategias de intervención para el PLIS-TdN}

Fotografía 4 - Ejemplo del esquema de intervención en un fragmento de territorio del PLIS-TdN

Fuente: PGT-I de las Tierras de los Navigli. Suministrado por el autor

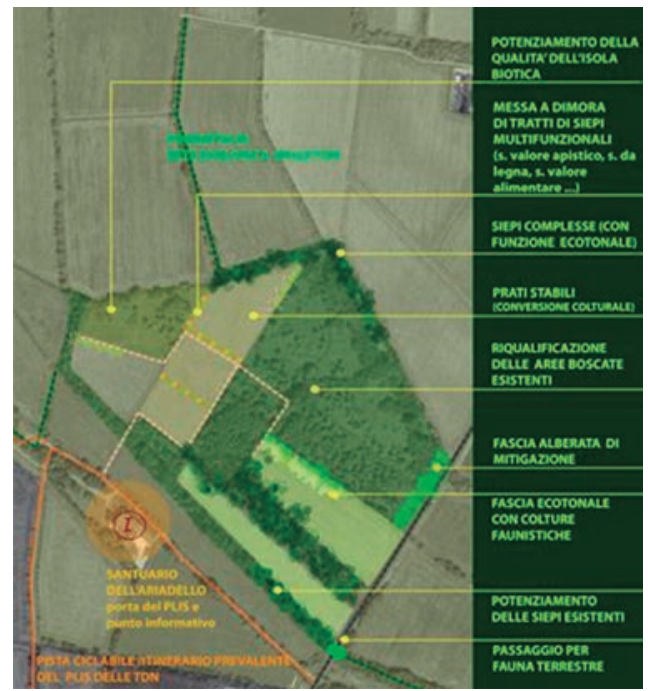

Las estrategias para conseguir los objetivos del PLIS-TdN han sido elaboradas con base a dos principios: la transversalidad y la integración de proyectos individuales, los cuales deben siempre concurrir para alcanzar los objetivos generales y declinar más de una estrategia al integrar los diferentes proyectos, la construcción de sinergias en la orientación de los procesos territoriales finalizados en la valorización del PLIS-TdN y la integración del PLIS$\mathrm{TdN}$ en el más amplio territorio de las $\mathrm{TdN}$.

Las estrategias para mejorar la calidad difusa del paisaje se integran con las de valorización del sistema productivo agrícola y están dirigidas a la gestión armónica de la transformación del paisaje (Fotografía 4). Son previstos servicios de tipo recreativo, cultural y dedicados a la producción agrícola para integrar la oferta de bienes y de servicios producidos en el PLIS-TdN con las exigencias de los potenciales usuarios y/o consumidores $y$, así mismo, especializar los servicios respecto a las líneas de producción cimentadas sobre factores típicos del área, prácticas agrícolas innovadoras e iniciativas de tipo recreativo y deportivo.

Además, la difusión de plantas de producción de energía a través de la utilización de las aguas residuales provenientes de criaderos zootécnicos y de residuos de la producción agrícola permite una recuperación de energía proveniente de los materiales de residuo y paralelamente, la reducción de los problemas que derivan de la contaminación por nitratos y de los malos olores. 
El proyecto de red ecológica constituye la referencia para la individuación y la sucesiva activación de intervenciones como el fortalecimiento de la vegetación ribereña, la protección y el mejoramiento de los bosques, la limitación de las rectificaciones agrarias funcionales, la eliminación de las discontinuidades altimétricas, la protección integral y la recuperación del sistema de irrigación, la valorización de los molinos, el desarrollo de nuevas líneas de diseño del paisaje agrario con base en métodos de cultivos biológicos, la incentivación de la forestación de los terrenos agrícolas abandonados, la protección y la recuperación de los edificios rurales, la incentivación y el desarrollo de nuevas tipologías constructivas para las plantas al servicio de la agricultura (invernaderos, silos, graneros, granjas y otras) para garantizar una buena inserción en el ambiente y en el paisaje. Además, han sido definidas las directrices sobre la gestión de las transformaciones territoriales que inciden sobre los elementos de la red ecológica como, por ejemplo, los proyectos de infraestructura que atraviesan los corredores ecológicos.

Los criterios para la conservación y la transformación de los núcleos rurales existentes apuntan a conservar de manera rigurosa las porciones de edificios rurales históricos y de valor histórico-arquitectónico, mientras la posibilidad de desempeñar nuevas funciones y actividades en los núcleos rurales abandonados, sin valor, deben ser siempre coherentes con las actividades agrícolas presentes en el núcleo individual y en el entorno. Finalmente, la conversión residencial de los núcleos totalmente abandonados, sólo es admisible si las viejas granjas (cascine) son accesibles desde las carreteras principales y son compatibles con la presencia de otras actividades agrícolas.

Los nuevos edificios rurales pueden ser localizados sólo en inmediaciones de los núcleos ya existentes o, si no es posible, al margen de las carreteras campestres. Estos nuevos edificios deben proporcionar volúmenes articulados sobre los terrenos para no crear frentes y masas impactantes, utilizar para las cubiertas y las fachadas, materiales similares, en términos de color y percepción, a las cubiertas y a las fachadas tradicionales.

Los edificios rurales y las construcciones de baja calidad y en mal estado de conservación, van mitigados preferiblemente a través de intervenciones de manutención y de recuperación, o de lo contrario, con intervenciones de camuflaje (blindaje) con hileras de árboles o verde vertical, mientras que las fábricas u otras instalaciones pueden ser mitigados valiéndose del enterramiento parcial y del blindaje verde. Por otra parte, si están bien proyectados y mantenidos, las instalaciones tecnológicas constituyen los elementos de valor paisajístico, mientras otras en desuso y en estado de abandono, como los depósitos de materiales residuales deben ser removidas.

Un aspecto fundamental para la cualificación territorial y paisajística del PLIS-TdN es la correcta planificación de sus márgenes, los cuales pueden interesar las áreas urbanas, los ámbitos productivos, las carreteras existentes y de proyecto, las áreas de alto interés de lo paisajístico-ambiental y las áreas de expansión destinadas a nuevos asentamientos. Para todas estas situaciones han sido individuadas intervenciones funcionales específicas y relaciones de proximidad, como por ejemplo la relación directa entre tejidos urbanos y áreas de parques, la eliminación o la mitigación de las criticidades presentes, sea de tipo perceptivo, en el caso de edificaciones o actividades incongruentes con los caracteres paisajísticos y funcionales, en el caso de actividades incongruentes con los objetivos del PLIS-TdN.

La movilidad, en sus más variadas formas, es considerada un elemento de soporte a la fruición del PLIS, que debe ser armonizada con el paisaje rural. Han sido definidos los criterios con los cuales proyectar las transformaciones y las intervenciones específicas. 
Fotografía 5 - Esquema de intervención para la recualificación de una infraestructura vial existente

Fuente: PGT-I de las Tierras del los Navigli. Suministrado por el autor.
Primero que todo, se debe cualificar la relación entre las áreas PLIS con las infraestructuras (carreteras y líneas ferroviarias, existentes y programadas) que le atraviesan o la interesan. Ya que el paisaje rural es un paisaje abierto en el cual son reconocibles tanto la estructura como los hitos (campaniles, granjas históricas, silos y los demás), es decir, los elementos que permiten la lectura y, por consiguiente, permiten percibir el "hermoso paisaje", en la proyección de itinerarios, infraestructuras de carreteras y servicios viene valorada la visual de los caracteres constitutivos del paisaje y mitigada la visual de los fenómenos detractores (Fotografía 5).

Además, para mejorar la accesibilidad de las áreas del PLIS-TdN desde el contexto local y territorial de referencia, vienen previstas una serie de intervenciones para realizarse progresivamente, y basadas sobre una clara "señalización" de los itinerarios para alcanzar dichas áreas y la evidenciarían de sus "puertas”, que son los lugares privilegiados de acceso al parque y por lo tanto, susceptibles de ser equipados.

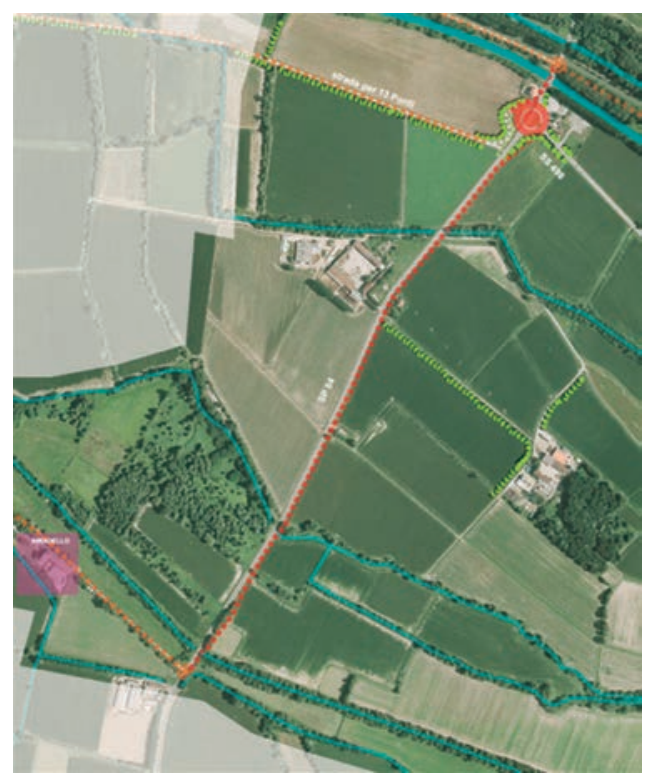

Fundamentalmente es la cualificación de las formas de movilidad en las áreas del parque y, por ende, la trama vial existente debe servir a las fincas agrícolas y de manera integrada a las funciones lúdico-recreativas. Al respecto, está previsto el refuerzo de la red ciclo-peatonal acompañada por un aumento de la seguridad en las carreteras, por la continuidad física y funcional, por la calidad de la construcción y por la capacidad de inervar el territorio y de injertarse en los centros urbanos. Además, el contexto espacial de los distintos itinerarios es objeto de cualificación a través de intervenciones especificas, ya sea de tipo ambiental (corredores ecológicos, equipamiento vegetaly otros) como también urbanístico-territorial (cualificación de los frentes edilicios de las áreas de limite urbano, muros verdes, integración con áreas lúdico-recreativas y demás.).

La capacidad de poner en marcha las estrategias previstas por el PLIS-TdN está dada por un conjunto de instrumentos de características diferentes y complementarias, que son:

- Los planes urbanísticos y los relativos instrumentos normativos y reguladores

- Las líneas guía y el ábaco de las intervenciones para la gestión de las transformaciones territoriales y paisajísticas, soporte de la proyectación de las intervenciones 
- Los instrumentos de gestión del PLIS, como los Reglamentos de uso, que consienten una mayor capacidad de incidir sobre las relaciones entre las actividades productivas y territorio, ambiente y paisaje y el Programa plurianual de las intervenciones que gestiona las intervenciones por realizarse en el marco de su validez temporal, que indican los recursos financieros y las modalidades de financiamiento.

- La cuenta ecológica, de la cual adquirir parte de los recursos económicos para realizar las intervenciones de mejoramiento ecológico. Tales recursos vendrán de la aplicación de la Compensación Ecológica Preventiva, que es un instrumento basado en el principio por el cual cada nueva edificación implica un impacto ecológico que va oportunamente compensado. En las Tierras de los Navigli, el mecanismo está basado sobre la monetización de las compensaciones relativas al conjunto de las intervenciones de transformación territorial, las cuales van atribuidas en medida proporcional al tipo y a la medida de la misma intervención, dando mayor importancia a las de expansión respecto a las de incremento volumétrico.

- Las mesas con las partes interesadas y con la ciudadanía, en manera de mantener en el tiempo, la participación de la población local.

\section{BIBLIOGRAFÍA}

BOERI S. y Lanzani A., Marini E. Il territorio che cambia. Ambienti e paesaggi della regione milanese. Abitare Segesta, Milano, 1993.

CAVALLINA, G. II margine inesistente. Alinea. Firenze, 1999.

INGERSOLL, R. Sprawltown, Meltemi, Roma, 2004.

DONADIEU P. Campagne urbane. Una nuova proposta di paesaggio della città. Editore Donzelli. Roma, 2006.

FREGOLENT L., Governare la dispersione. Franco Angeli Editore. Milano, 2005.

GAMBINO R. Conservare, innovare: paesaggio, ambiente e territorio, UTET, Torino, 1997.

GHIRRI, L. y POMPEO, Fabbri G. Natura e cultura del paesaggio agrario. Cittàstudi Edizioni, Milano, 1997.

PEANO, A. Il paesaggio nel futuro del mondo rurale. Esperienze e riflessioni sul territorio torinese. Alinea Editrice, Firenze, 2006.

PEANO, A. y VOGHERA, A. "Teoria e pratiche a partire dalla Convenzione Europea del Paesaggio / Theory and practices from the European Landscape Convention”. En: Architettura del Paesaggio. Vol. 16. 2007.

TURRI, E. La Megalopoli Padana. Marsilio. Venezia, 2000.

WALDHEIM, C. The Landscape Urbanism Reader. Princeton Architectural Press. New York, 2006. 